\title{
Filogenia y herencia arcaica en la obra de Freud: La búsqueda de la etiologia y la pasión por lo real
}

\author{
Silvana Vetö Honorato*1 \\ Mauro Vallejo*2
}

La obra teórica de Sigmund Freud contiene múltiples referencias al tópico de la herencia arcaica y la filogenia. El objetivo de este artículo es analizar ese aspecto de su obra, con el cometido de señalar las limitaciones de las interpretaciones más frecuentes, que tienden a ver alli un impacto del darwinismo en Freud o de simplemente negar su relevancia. Intentaremos mostrar que el recurso a la filogenia respondió enteramente a un interrogante clínico por la etiología de las neurosis.

Palabras clave: Filogenia, herencia arcaica, etiología, historia

\footnotetext{
${ }^{* 1}$ Universidad Andres Bello (Santiago, Chile)

*2 Universidad de Buenos Aires (Buenos Aires, Argentina)
} 


\section{ARTIGOS}

\section{El malentendido de la filogenia freudiana}

Toda indagación por el concepto de historia en Freud choca con el concepto de filogenia y, por esa vía, con la influencia directa de las teorías evolutivas decimonónicas, especialmente lamarckianas, darwinianas y haeckelianas, que los psicoanalistas han querido alejar de sí. Así lo sostuvo el psicoanalista austríaco Ernst Kris en el documental de la BBC realizado para el centenario del nacimiento de Freud (1956): "Las propensiones lamarckianas de Freud eran muy lamentadas por muchos de nosotros". (citado en Ritvo, 1990, p. 31; traducción propia)

Esas propensiones de Freud no cejaron nunca. Tan tardíamente como 1938, en "Moisés y la religión monoteísta", Freud (1939[193448]/1998m) escribía:

Ante una meditación más ceñida, no podemos sino confesarnos que desde hace tiempo nos comportamos como si la herencia de huellas mnémicas de lo vivenciado por los antepasados, independientemente de su comunicación directa o del influjo de la educación por ejemplo, estuviera fuera de cuestión. (...) Nosotros no podemos prescindir de este factor en el desarrollo biológico. (p. 96)

Freud sostiene que la tesis lamarckiana de la herencia de los caracteres adquiridos y la tesis de la recapitulación de la filogenia en la ontogenia, son fundamentos de sus planteamientos. A lo largo de este artículo haremos pie en ese no-poder-prescindir de la filogenia confesado por Freud. Y lo haremos en base a una serie de interrogantes que, por extraño que resulte, han sido descuidados por la mayoría de los estudios acerca de la obra freudiana: ¿para qué precisaba la filogenia (al punto de no poder renunciar a ella)?, 


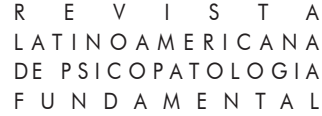

y sobre todo ¿a partir de cuándo el saber psicoanalítico sintió esa necesidad apremiante?

El tópico de la filogenia ha sido muy trabajado por la literatura especializada. Siendo un tanto esquemáticos, podemos aseverar que las lecturas tradicionales pueden ser divididas en tres grandes grupos. De un lado tenemos a los propios psicoanalistas, sobre todo a sus herederos inmediatos, quienes se vieron colocados en la tarea de defender el legado de su maestro. Respecto del tópico de la filogenia, la postura que asumieron consistió o bien en negar su relevancia al interior del opus freudiano, o bien en lamentar la presencia de ese punto incómodo, cual si se tratase meramente de un síntoma de la obcecación del maestro (Jones, 1953). De otro lado podríamos colocar a los historiadores más críticos, que a partir de la década de 1970 construyeron visiones muy documentadas y detalladas sobre el pasado de la disciplina. En algunos de esos recuentos, el análisis de la presencia de la filogenia en Freud servía al cometido iconoclasta de resaltar el parentesco estrecho entre la teoría freudiana y argumentos e hipótesis del siglo XIX, ya perimidos y obsoletos para comienzos de la última centuria (Sulloway, 1979). Por último cabría ubicar las investigaciones universitarias más desapasionadas, que sin estab-

546 lecer juicios valorativos sobre esas continuidades, han captado en la adhesión freudiana a la filogenia un síntoma de la inscripción de ese saber en la cultura científica de fines de siglo (Ritvo, 1990; Otis, 1994; Duvernay Bolens, 2001).

La sola existencia de tan variados y numerosos ensayos acerca de la filogenia freudiana hace justicia a un punto que casi todos los estudiosos han remarcado de modo certero: a diferencia de otros enunciados polémicos o abandonados luego por la ortodoxia psicoanalítica — por ejemplo, la furtiva esperanza de Freud de hallar en la química el secreto de la sexualidad humana -, los argumentos freudianos sobre el pasado de la humanidad son no solamente insistentes, sino que además remiten a tópicos esenciales de su edificio teórico. Ahora bien, igual de justo es afirmar que restan aún muchas preguntas por responder en lo referido a esta problemática. Más aún, según nuestro parecer los ensayos existentes son incapaces de afrontar el interrogante más esencial, referido al motivo por el cual Freud apeló a la filogenia a partir de cierto momento específico.

El presente artículo consta de dos secciones. En la primera de ellas, intentaremos documentar el modo en que Freud efectuó conjeturas sobre el pasado filogenético, prestando especial atención a la manera en que aquellas se articulaban con otros conceptos fundamentales del psicoanálisis, e indicando la relevancia de algunas fuentes no tenidas en consideración por los 


\section{ARTIGOS}

comentadores. Nos concentraremos casi exclusivamente en fragmentos publicados por Freud, aludiendo sólo en contadas ocasiones a sus intervenciones en la Sociedad Psicoanalítica de Viena. A sus correspondencias y a los borradores exhumados luego de su muerte, haremos referencia en menos ocasiones aún, pues de lo contrario nos enfrentaríamos con evidencias documentales demasiado numerosas.

En la sección final de este escrito desplegaremos nuestra hipótesis respecto de aquel interrogante esencial. Ello nos permitirá circunscribir las limitaciones de la lectura más difundida (que ve en la filogenia freudiana un mero síntoma del credo biologicista o darwiniano del autor), y al mismo tiempo estaremos en condiciones de reintegrar el interés por la filogenia en el registro clínico al que siempre perteneció.

\section{Conjeturas sobre el pasado filogenético, o la imaginación histórica de Freud}

La primera vez que Freud alude a la filogenia en sus escritos publicados es en 1911, en el párrafo final de su Apéndice al trabajo sobre el Presidente Schreber. Allí, y en referencia a la tesis de Jung sobre la persistencia en los neuróticos de las viejas potencias mitopoyéticas de la humanidad, Freud afirma que muy pronto sería posible agregar "el complemento antropológico, de concepción filogenética" a una conjetura referida la ontogenia:

Hemos dicho: "En la neurosis y en el sueño reencontramos al niño, con las propiedades de sus modos de pensar y de su vida afectiva". Completaremos: "También hallamos al hombre salvaje, primitivo, tal como él se nos muestra a la luz de la arqueología y de la etnología". (Freud, 1911[1910]/1998e, p. 76; énfasis del original)

El "salvaje, primitivo", en palabras de Freud, tienen su paralelo ontogenético en el niño, y así, la neurosis y el sueño, como las otras formaciones del inconsciente son resultado de dichas potencias creadoras de la filogénesis, recapituladas en el desarrollo ontogenético individual.

Del mismo modo, en un fragmento agregado en 1919 al capítulo VII de La interpretación de los sueños, Freud (1900[1899]/1998a) afirma que el soñar es una regresión a la infancia del soñante, tras la cual "se nos promete también alcanzar una perspectiva sobre la infancia filogenética, sobre el desarrollo del género humano, del cual el del individuo es de hecho una repetición abreviada, influida por las circunstancias contingentes de su vida" (p. 542). 


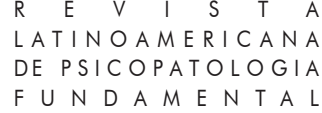

El Apéndice de 1911 señala, entonces, el inicio de una línea argumentativa que irá desplegándose con el correr de los años. No estamos, empero, ante la más temprana ocasión en que razonamientos ligados a la filogenia habían ocupado al psicoanálisis, o a Freud en particular. En efecto, cabría indicar que en las reuniones mantenidas por Freud y sus colegas vieneses aquella temática había sido debatida con anterioridad. Ya en marzo de 1908 Fritz Wittels había realizado una exposición sobre los avatares atravesados por la humanidad en la época de las glaciaciones, y sobre el modo en que, transmisión filogenética mediante, esos accidentes habían repercutido en el destino ulterior de la humanidad (Numberg \& Federn, 1962; Vallejo, 2008). Esta fuente testimonia que en esa misma época, y de manera contemporánea al escrito sobre Schreber, cobra forma una hipótesis que ocupará un lugar destacado en el razonamiento freudiano acerca de la herencia arcaica. Nos referimos al postulado según el cual los impulsos, fantasías o represiones que en el hombre actual despiertan espontáneamente de su interior, fueron alguna vez exigencias o experiencias provenientes del afuera. Por ejemplo, en marzo de 1909 el creador del psicoanálisis aventura que la fantasía incestuosa es un "rasgo arcaico" de los neuróticos, pues en el pasado remoto de 548 la humanidad la ligazón endogámica había sido una realidad efectiva (Federn y Numberg, 1967). En unas notas redactadas a fines de 1911, Freud afirmó: "Todos los impedimentos internos fueron anteriormente resistencias exteriores — Explicación filogenética de la represión" (Grubrich-Simitis, 1997, p. 118). Más abajo veremos que esas ideas acerca del origen filogenético de las represiones y los impulsos constituyen una columna vertebral del ensayo sobre "Tótem y tabú", publicado en 1913.

En 1915-16, en su conferencias titulada "Rasgos arcaicos e infantilismo en el sueño", Freud (1916[1915-16]/1998g) retoma los planteamientos de 1911. En el sueño, afirma, se pone en juego un "modo de expresión" que nos resulta incomprensible, puesto que se "remonta a estados de nuestro desarrollo intelectual superados ha mucho por nosotros": el "lenguaje figural", la "referencia simbólica" (p. 182), que habrían existido antes del desarrollo de lo que Freud llama allí "lenguaje discursivo". Se trata entonces, añade, de un modo de expresión, arcaico o regresivo. Pero no se trata únicamente de una forma de expresión abandonada en el curso de la ontogénesis, sino también de la filogénesis:

La prehistoria a que el trabajo del sueño nos reconduce es doble: en primer lugar, a la prehistoria individual, la infancia; y por otra parte, en la medida en que cada individuo repite abreviadamente en su infancia, de alguna manera, el 


\section{ARTIGOS}

desarrollo todo de la especie humana, también esta otra prehistoria, la filogenética. (...) Así, me parece, la referencia simbólica, que el individuo en ningún caso aprendió, tiene justificado derecho a que se la considere una herencia filogenética. (Freud, 1916[1915-16]/1998g, p. 182)

Freud establece una división entre historia y prehistoria, marcada aquí por el inicio de la palabra. La prehistoria individual, cuando el niño aun no puede hablar; la prehistoria de la especie, cuando los primitivos figuraban sus deseos y necesidades en símbolos en las paredes. ${ }^{1}$ No son modalidades de expresión ni referencias simbólicas perimidas, olvidadas, sino siempre actuales, insistentes, que retornan perturbando el presente. La regresión es el mecanismo que hace re-aparecer en escena a ese pasado, a esa prehistoria.

De la misma manera, aunque con un mayor resguardo, explica Freud en 1918 en el historial del "hombre de los lobos" una serie de escenas de la vida infantil:

Las escenas de observación del comercio sexual entre los padres, de seducción en la infancia y de amenaza de castración son indudablemente un patrimonio heredado, herencia filogenética, pero también pueden ser adquisición del vivenciar individual. (... ) en la historia primordial de las neurosis vemos que el niño echa mano de esa vivencia filogenética toda vez que su propio vivenciar no basta. Llena las lagunas de la verdad individual con una verdad prehistórica, pone la experiencia de los ancestros en el lugar de la propia. (Freud, 1918[1914]/1998i, p. 89)

En 1919, en "Pegan a un niño", Freud (1919/1998j) establece una definición bien poco citada de lo inconsciente, que va en la misma línea de lo que estamos intentando desentrañar: "El núcleo de lo inconsciente anímico lo constituye la herencia arcaica del ser humano, y de ella sucumbe al proceso represivo todo cuanto en el progreso hacia fases evolutivas posteriores, debe ser relegado por inconciliable con lo nuevo y perjudicial para él" (p. 199). Tal como se lee: para Freud, el "núcleo de lo inconsciente" lo constituye esta herencia arcaica.

\footnotetext{
${ }^{1}$ La conjetura sobre un pasado remoto donde las capacidades simbólicas escaseaban, figura en el centro argumental del escrito en que Freud lleva al extremo su interés por la herencia arcaica y la filogenia. Hacemos alusión al manuscrito enviado a Ferenczi el 28 de julio de 1915 (Grubrich-Simitis, 1985). Un análisis detenido de esas páginas excede el cometido de este artículo. Por otro lado, las cartas intercambiadas por Freud y Ferenczi en ese período (1915-1916) están repletas de alusiones al tópico de la filogenia (Bravant \& Falzeder, 2001).
} 
En su vigésimo segunda conferencia de introducción al psicoanálisis, Freud (1917[1916-17/1998h) alude al desarrollo diferencial del yo y de la libido, sosteniendo que "ambos son en el fondo heredados, unas repeticiones abreviadas de la evolución que la humanidad toda ha recorrido desde sus épocas originarias y por lapsos prolongadísimos" (p. 322). Si bien agrega que este "origen filogenético" se ve más fácilmente en el desarrollo libidinal, afirma también que en el ser humano dicho origen está velado "por la circunstancia de que algo en el fondo heredado es, empero, vuelto a adquirir en el desarrollo individual" (p. 323). Las mismas circunstancias, dice, que en los tiempos primordiales impusieron una adquisición, seguirían activas sobre el individuo. "Yo diría que en ese tiempo operó como una creación, y ahora actúa como un llamado" (p. 323): el llamado filogenético.

Según esta idea, Freud derriba, a decir verdad, la oposición clásica entre lo hereditario y lo adquirido, puesto que sostiene que aquello que se habría heredado, una predisposición, fue antiguamente adquirida bajo determinadas circunstancias: "Las disposiciones constitucionales son, con seguridad, la secuela que dejaron las vivencias de nuestros antepasados; también ellas se adquirieron una vez: sin tal adquisición no habría herencia alguna" (p. 329). ${ }^{2}$

Ahora bien, es en "Tótem y tabú", publicado en 1913, donde Freud (1913[1912-13]/1998f) imagina la coyuntura en cual el ser humano habría adquirido su patrimonio hereditario, vale decir, el mito de adquisición de esa herencia arcaica que llamamos lo inconsciente. Este mito se sostiene en la existencia y represión de las dos pulsiones primordiales del ser humano, que explican a su vez la universalidad del Complejo de Edipo, y en base a las cuales Freud imagina se habría creado la sociedad, la cultura, las religiones. De hecho, es en ese texto en el cual la importancia del entramado onto-filogenético muestra su mayor eficacia, y la herencia arcaica su estatuto de fundamento. Allí Freud (1913[1912-13]/1998f) señala, a propósito de la crítica a la oposición adquirido-heredado que venimos interrogando:

Los tabúes serían unas prohibiciones antiquísimas, impuestas en su tiempo desde afuera a una generación de hombres primitivos, o sea: una generación anterior se los inculcó con violencia. Tales prohibiciones recayeron sobre actividades hacia las que había fuerte inclinación. Luego se conservaron de generación en generación, acaso por mero efecto de la tradición sustentada por

${ }^{2}$ Freud retomará esa misma hipótesis en otro escrito (Freud, 1998I, p. 242). 


\section{ARTIGOS}

la autoridad parental y social. Pero también es posible que se "organizaran" ya dentro de las organizaciones posteriores como una pieza de patrimonio psíquico heredado. (p. 39)

Ahora bien, la escena en que dichas prohibiciones habrían sido inculcadas con violencia, es bien conocida. Se trata de lo que Freud llama "deducción histórico-conjetural", la cual establece un nexo entre la hipótesis de Charles Darwin y James J. Atkinson, para sostener que el hombre primordial se organizaba en hordas pequeñas en torno a un macho viejo, fuerte y celoso, que prohibía las relaciones sexuales al interior de la horda, junto a las tesis de William Robertson Smith acerca del banquete totémico sacrificial.

En "Moisés y la religión monoteísta", publicado en 1938, Freud (1939[1934-48]/1998m) otorga nuevas elucidaciones respecto de estos mismos problemas, las cuales terminan por dejar a Freud en una zona espesa, y aún más opaca, a ojos de sus discípulos. El parricidio primordial fue el primer trauma de la humanidad, sus huellas habrían sido reprimidas imprimiendo fijaciones sobre las que habrá de volver la regresión. Respecto de las "impresiones de los traumas tempranos", afirma que esta hipótesis no acarrea ninguna complicación, pero que cuando se trata de aquellos contenidos que no pudieron ser vivenciados por el propio individuo, sino "aportados con el nacimiento, fragmentos de origen filogenético, una herencia arcaica" (Freud, 1939[1934-48]/1998m, p. 94), aparecen nuevas dificultades.

Plantea Freud que la herencia arcaica sería un factor constitucional, que no contiene sólo predisposiciones, sino también "contenidos, huellas mnémicas de lo vivenciado por generaciones anteriores" (Freud, 1939[1934-48]/1998m, p. 96). Las reacciones de los niños frente a sucesos traumáticos, sostiene, nos sorprenden por cuanto "no se atienen de manera estricta a lo real y efectivamente vivenciado por sí-mismo, sino que se distancian de esto de una manera que se adecúa mucho más al modelo de un suceso filogenético $\mathrm{y}$, en términos universales, sólo en virtud de su influjo se pueden explicar" (p. 95). Así funcionarían el complejo de Edipo y el complejo de Castración. De este modo, afirma lo que llama una "temeridad inevitable" (p. 96): "los seres humanos han sabido siempre 'de aquella particular manera' que antaño poseyeron un padre primordial y lo mataron" (p. 97). La condición que un saber tal debe cumplir para transmitirse de generación en generación sin

${ }^{3}$ Dicha tesis muy pronto sería insertada en la obra fundamental sobre la sexualidad (Freud, $1905 / 1998$ b, p. 147 n.). 
sucumbir al rechazo que ejercería sobre él el juicio y la razón, es que haya sido reprimido, para que así, desde su estatuto de fijación inconsciente, su retorno pueda desplegar efectos tan potentes e indesafiables. Desde ese estatuto de reprimido, producto de lo cual se vuelve fundamento, el asesinato del padre operaría como un "saber-no-sabido" actual para cada individuo, que actuaría, según se establece luego de "El yo y el ello" (1923/1998k), por medio del arma más efectiva de nuestra herencia arcaica: el superyó y su sentimiento inconsciente de culpa. Así, en el "Esquema del psicoanálisis", Freud (1940[1938]/1998n) afirma:

Una parte de las conquistas culturales sin duda ha dejado como secuela su precipitado dentro del ello, mucho de lo que el superyó trae despertará un eco en el ello, y no poco de lo que el niño vivencia como nuevo experimentará un refuerzo porque repite un ancestral vivenciar filogenético. (...) De este modo, el superyó ocupa una suerte de posición media entre ello y mundo exterior, reúne en sí los influjos del presente y el pasado. En la institución del superyó uno vivencia, digamos así, un ejemplo del modo en que el presente es traspuesto en pasado. (...) (pp. 208-9)

\section{A modo de conclusión: límites de las tesis de la influencia y avance de la hipótesis clínica}

Hemos intentado documentar la arraigada apelación por parte de Freud a razonamientos de tenor filogenético. Hemos comprobado, en tal sentido, que aproximadamente a partir de 1910 Freud recurrió a argumentaciones sobre el pasado de la humanidad de modo sostenido y razonado. Más aún, pretendió hallar en la filogenia respuestas, o esbozos de respuestas, para preguntas que resultaban fundamentales para su empresa teórica: la génesis de la represión, la esencia de las fantasías primordiales, el núcleo del inconsciente, el origen de la cultura, entre otras.

Gran parte de los comentadores han visto en todo ello la evidencia incuestionable del impacto que el pensamiento evolucionista y biológico tuvo sobre el creador del psicoanálisis (Sulloway, 1979; Assoun, 1981; Grubrich-Simitis, 1985; Otis, 1994; Duvernay Bolens, 2001). Ese diagnóstico es certero, pero también insuficiente, sobre todo porque es incapaz de hacer frente a los interrogantes más esenciales. El hecho de señalar una filiación darwiniana en Freud no arroja pistas sobre la función particular que la filogenia desempeñó en aquel entramado teórico, y menos aún sobre la razón por la cual la herencia arcaica 


\section{ARTIGOS}

cobró relieve recién alrededor de 1910, cuando ya estaban colocados varios de los pilares de la doctrina psicoanalítica. De hecho, si la tesis de la influencia biologicista fuera convincente, ¿no deberíamos notar un impacto mayor de ese pasado cuanto más cerca estemos de los inicios del psicoanálisis?

Según nuestro parecer, a los fines de despejar estos enigmas, es menester tomar en consideración algunos elementos clave de la apuesta intelectual de Freud. En esta conclusión no haremos más que dejar asentados algunos planteos, que pueden quizá funcionar como catalizadores de futuras indagaciones. Más de una vez se ha señalado que el psicoanálisis, en tanto discurso innovador, nace en el momento en que Freud puede romper con la tradición recibida, que en el terreno de la neuropatología era equiparable con la teoría de la heredo-degeneración. En efecto, en la medicina anterior a Freud el problema de lo histórico y del pasado gozaba ya de un marcado protagonismo. Las patologías eran, para decirlo esquemáticamente, la derivación actual de una línea de tiempo ligada a la estirpe o la sangre (Coffin, 2003). Freud conoció de cerca ese paradigma, pues se formó en él, y siguiendo a su maestro J.-M. Charcot, lo hizo suyo al comienzo de su recorrido (Andersson, 1962; Gilman, 1993). Pudo romper con ese modo de pensar cuando contó con las herramientas para conjugar de otra manera la problemática histórico-temporal. $\mathrm{Su}$ abandono de la teoría hereditaria, de hecho, fue consustancial al hallazgo de una fórmula etiológica que emulaba la revolución médica auspiciada por el descubrimiento de Pasteur (Carter, 1980). Los rudimentos del psicoanálisis emergen entre 1893 y 1895 porque en ese instante Freud dedica todas sus energías a perfilar una definición estrictamente etiológica de las enfermedades. En esos años, su búsqueda se encamina hacia etiologías específicas, y no ya hacia orígenes sanguíneos dudosos. El problema del trauma vivenciado, que en Charcot quedaba parcialmente diluido en una doctrina hereditarista en que la causa última nunca era el verdadero interrogante, fue a partir de entonces el engranaje esencial de un discurso que se obstinó en aprehender en la génesis histórica la esencia de lo humano.

Siguiendo un filo historicista que, en parte gracias al darwinismo, tiñó tan fuertemente a las ciencias sociales de fines de siglo, en Freud lo humano fue siempre un problema de génesis. El primer desarrollo del pensamiento psicoanalítico puede ser traducido como la puesta en marcha de un historicismo aplicado en varios frentes. En el terreno de la patología - teoría de la seducción mediante - las enfermedades comenzaron a ser bosquejadas a partir de 1895 como el precipitado de vivencias que se remontaban la infancia más temprana (Vallejo, 2012). En el área del funcionamiento inconsciente, 
no sólo se postuló que los sueños, los chistes y los actos fallidos debían ser abordados en función de su proceso de creación, sino que también se trabajó bajo el planteamiento de que lo infantil era la matriz que gobernaba esas producciones: por un lado, porque las vivencias e impulsos de la infancia eran en última instancia el punto al que había que remontar las manifestaciones inconscientes, y por otro, porque el funcionamiento psíquico infantil, atrapado en su simbolismo y demás leyes, otorgaba la clave con la cual leer esos fenómenos humanos. Por último, en el escabroso terreno de la sexualidad, elevado al estatuto de motor primordial de intereses y deseos, lo infantil fue asimismo colocado en el escalafón de lo instituyente y ordenador.

Ese recurso transparente a la historia ordenaba el pensamiento de Freud hasta la primera década del siglo XX. Pero luego de la caída de la teoría de la seducción, a fines de 1897, ese proyecto se había quedado sin una pieza clave: la explicación patológica. En efecto, los avances en el terreno de las formaciones del inconsciente se habían realizado en desmedro de una fundamentación alternativa de lo enfermo. Podemos trazar ese recorrido mediante la mención a obras capitales: si el término "psicoanálisis" emerge en 1896 en el seno de uno de los escritos de la teoría de la seducción - esto es, emerge 554 en un momento en que todo el pensamiento de Freud se direcciona hacia una definición genética de lo patológico, centrada en la potencialidad de los recuerdos inconscientes de la infancia -, la doctrina recibe su verdadero andamiaje conceptual en los años de transición en que Freud ha renunciado al intento de hallar un nuevo esclarecimiento causal de las enfermedades, es decir, entre 1899 y 1905, La interpretación de los sueños y "Tres ensayos de teoría sexual". Durante todo ese tiempo, y en flagrante contraposición a su período anterior, Freud parece abandonar el terreno de la psicopatología. La excepción está dada por el caso Dora, elaborado en 1901, pero publicado cuatro años más tarde. De todas maneras, tal y como ha desarrollado Georges Makari (1997), ese historial queda atrapado en esta etapa de transición, en la cual se echa de menos una hipótesis clara y decidida sobre el origen de la enfermedad. Habrá que aguardar al cambio de década (con Hans y el Hombre de las Ratas) para que la pluma freudiana vuelva a internarse de lleno en las arenas de la patología. Recién por ese entonces, gracias a sus nuevas intelecciones sobre los impulsos sexuales, las fantasías inconscientes, y un poco más tarde la figura del Edipo, Freud vuelve a ensayar una explicación novedosa sobre las enfermedades nerviosas. $Y$ es entonces que apela a los argumentos de la filogenia. Ellos estaban destinados a suplir los vacíos y las vacilaciones de la nueva teoría clínica. 


\section{ARTIGOS}

¿Qué le pide Freud a la filogenia? Las citas lo dicen a viva voz. Le solicita que responda, en última instancia, por la esencia genética de los nuevos engranajes de la teoría psicopatológica: el pasado de la humanidad ha de responder por el origen de las represiones, el origen de las fantasías primordiales, por la causa de la sexualidad en dos tiempos etc.

Si nuestra lectura es correcta, la centralidad de la filogenia en Freud no ha de ser tomada ni como una retardada influencia de marcos darwinianos fuera de moda, y menos aún como un objeto extraño dentro de la teoría. La emergencia del interés por la herencia arcaica fue una derivación contingente de un proyecto intelectual que desde el inicio quiso traducir lo humano en términos de su génesis histórica, sobre todo en el dominio de lo disfuncional. Más aún, una última observación apunta a enfatizar que el recurso a la filogenia se adecuó en Freud a una lógica perenne. Si Freud recurrió a la especulación sobre el pasado de la humanidad en aras de sortear interrogantes esenciales, ello se debió a que esperaba encontrar allí el único tipo de conjeturas que su ideario aceptaba. Cabe hablar aquí de su pasión por lo real. La apelación a la filogenia fue el postrero intento de reenviar lo humano a la operatoria de un pasado real. Contrariamente a lo que suponen algunas lecturas consensuadas, según las cuales tras la caída de la seducción (1897) Freud desechó el cometido de hallar escenas o episodios en el pasado real y fechable, es posible afirmar que la creciente atención prestada al mundo de las fantasías, o la insistente alusión a una temporalidad de la Nachträglichkeit, nunca conllevó la renuncia a la tarea de circunscribir momentos o acontecimientos del pasado efectivo que fueran responsables de la constitución subjetiva. Pues bien, a esa obstinación se plegó la indagación sobre la herencia arcaica. Ella fue el más encarnizado ensayo de localizar, en un pasado ahora más remoto aún, el hecho real que desde su olvido productivo condiciona el presente.

\section{Referencias}

Andersson, O. (1962). Freud avant Freud. La préhistoire de la psychanalyse, 1886-1996. Paris, France: Les empêcheurs de penser en rond.

Assoun, P.-L. (1981). Introducción a la epistemología freudiana. México,México, D.F.: Siglo XXI.

Bravant, E.; Ernst, F., \& Giamperi-Deutsch, P. (eds.). (2001). Sigmund Freud-Sándor Ferenczi. Correspondencia completa. Madrid, España: Editorial Síntesis. 
Carter, C. (1980). Germ Theory, Hysteria, and Freud's early Work in Psychopathology. Medical History, 24, 259-274.

Coffin, J.-C. (2003). La transmission de la folie, 1850-1914. Paris, France: L'Harmattan.

Duvernay Bolens, J. (2001). La théorie de la récapitulation de Haeckel à Freud. Topique, 75, 13-34.

Freud, S. (1998a). La interpretación de los sueños. In Obras completas (Vol. IV y V). Buenos Aires, Argentina: Amorrortu. (Trabajo original publicado en 1900[1899]).

Freud, S. (1998b). Tres ensayos de teoría sexual. In Obras completas (vol. VII, pp. 1-221). Buenos Aires, Argentina: Amorrortu. (Trabajo original publicado en 1905).

Freud, S. (1998c). Fragmento de análisis de un caso de histeria. In Obras completas (Vol. VII, pp. 1-108). Buenos Aires, Argentina: Amorrortu. (Trabajo original publicado en 1905[1901]).

Freud, S. (1998e). Puntualizaciones psicoanalíticas sobre un caso de paranoia (Dementia paranoides) descrito autobiográficamente. In Obras completas (Vol. XII, pp. 1-76). Buenos Aires, Argentina: Amorrortu. (Trabajo original publicado en 1911[1910]).

Freud, S. (1998f). Tótem y tabú. Algunas concordancias de la vida anímica de los salvajes y de los neuróticos. In Obras completas, (Vol. XIII, pp. 1-161). Buenos Aires, Argentina: Amorrortu. (Trabajo original publicado en 1913[1912-13]).

Freud, S. (1998g). 13 ${ }^{\text {a }}$ Conferencia. Rasgos arcaicos e infantilismo en el sueño. In Obras completas (Vol. XV, pp. 182-194). Buenos Aires: Amorrortu. (Trabajo original publicado en 1916[1915-16]).

Freud, S. (1998h). $22^{\circ}$ conferencia. Algunas perspectivas sobre el desarrollo y la regresión. In Obras completas (Vol. XVI, pp. 309-325). Buenos Aires, Argentina: Amorrortu. (Trabajo original publicado en 1917[1916-17]).

Freud, S. (1998i). De la historia de una neurosis infantil. In Obras completas (Vol. XVII, pp. 1-110). Buenos Aires, Argentina: Amorrortu. (Trabajo original publicado en 1918[1914]).

Freud, S. (1998j). "Pegan a un niño". Contribución al conocimiento de la génesis de las perversiones sexuales. In Obras completas (Vol. XVII, pp. 173-200). Buenos Aires, Argentina: Amorrortu. (Trabajo original publicado en 1919).

Freud, S. (1998k). El yo y el ello. In Obras completas (Vol. XIX, pp. 1-66). Buenos Aires, Argentina: Amorrortu. (Trabajo original publicado en 1923).

Freud, S. (19981). Análisis terminable e interminable. In Obras completas (Vol. XXIII, pp. 211-254). Buenos Aires, Argentina: Amorrortu. (Trabajo original publicado en 1937).

Freud, S. (1998m). Moisés y la religión monoteísta. In Obras completas (Vol. XXIII, pp. 1-131). Buenos Aires, Argentina: Amorrortu. (Trabajo original publicado en 1939[1934-48]).

Freud, S. (1998n). Esquema del psicoanálisis. In Obras completas (Vol. XXIII, pp. 133-210). Buenos Aires, Argentina: Amorrortu. (Trabajo original publicado en 1940[1938]).

Gilman, S. (1993). The case of Sigmund Freud. Medicine and identity at the Fin de Siècle. Baltimore and London: The John Hopkins University Press.

Grubrich-Simitis, I. (1985). Metapsicología y metabiología. In I. Grubrich-Simitis (Ed.), Sinopsis de las neurosis de transferencia. Ensayo de metapsicología (pp. 87-121). Madrid, España: Ariel. 


\section{ARTIGOS}

Grubrich-Simitis, I. (1997). Volver a los textos de Freud. Madrid, España: Biblioteca Nueva. Jones, E. (1953). Vida y obra de Sigmund Freud. Buenos Aires, Argentina: Horme.

Makari, G. (1997). Dora's Hysteria and the Maturation of Sigmund Freud's Transference Theory: a New Historical Interpretation. Journal of the American Psychoanalityc Association, 45(4), 1061-1096.

Nunberg, H., \& Federn, E. (ed.) (1967). Minutes of the Vienna Psychoanalytic Society (Vol. I). New York, U.S.A.: International Universities Press.

Nunberg, H., \& Federn, E. (ed.) (1962). Minutes of the Vienna Psychoanalytic Society (Vol. II). New York, U.S.A.: International Universities Press.

Otis, L. (1994). Organic Memory: History and the Body in the Late Nineteenth and Early Twentieth Centuries. Lincoln, U.S.A.: University of Nevraska Press.

Ritvo, L. B. (1990). Darwin's Influence on Freud. A Tale of Two Sciences. New Haven, U.S.A.: Yale University Press.

Sulloway, F. (1979). Freud, Biologist of the Mind. Beyond the Psychoanalytic Legend. New York, U.S.A.: Basic Books.

Vallejo, M. (2008). Los miércoles por la noche, alrededor de Freud. Buenos Aires, Argentina: Letra Viva.

Vallejo, M. (2012). La seducción freudiana (1895-1897). Un ensayo de genética textual. Buenos Aires, Argentina: Letra Viva.

\section{Resúmenes}

(Filogenia e herança arcaica na obra de Freud: a busca da etiologia e a paixão pelo real)

O trabalho teórico de Sigmund Freud contém várias referências ao tema da herança arcaica, ou filogenia. O objetivo deste artigo é analisar esse aspecto de sua obra, com a tarefa de apontar as limitações das interpretações mais comuns, que tendem a ver um impacto do Darwinismo em Freud ou simplesmente negar sua relevância. Procuramos demonstrar que o uso da filogenia respondeu inteiramente a uma pergunta clínica sobre a etiologia da neurose.

Palavras-chave: Filogenia, herencia arcaica, etiología, história

(Phylogeny and archaic inheritance in the work of Freud: the quest for etiology and the passion for what is real)

The theoretical work of Sigmund Freud contains multiple references to the topic of archaic inheritance and phylogeny. The aim of this paper is to analyze this aspect of his work, in order to point out the limitations of its most frequent interpretations, which tend to perceive the impact of Darwinism on Freud, or simply deny its 


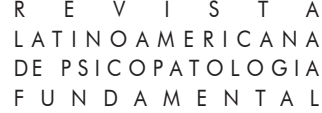

relevance. We intend to prove that using phylogeny responded entirely to a clinical question regarding the etiology of neuroses.

Key words: Phylogeny, archaic inheritance, etiology, history

(Phylogénie et héritage archaïque dans l'œuvre de Freud: la recherche de l'étiologie et la passion du réel)

Le travail théorique de Sigmund Freud contient plusieurs références au sujet de l'héritage archaïque ou de la phylogénie. Le but de cet article est d'analyser cet aspect de son travail, soit de souligner les limites des interprétations les plus courantes qui ont tendance à voir un impact du darwinisme chez Freud ou tout simplement à nier sa pertinence. Nous essayons ainsi de démontrer que l'utilisation de la phylogénie répondait parfaitement à la question clinique sur l'étiologie de la névrose.

Mots clés: Phylogénie, héritage archaïque, étiologie, histoire

(Phylogenie und archaische Erbe in Freuds Werk: die Suche nach der Ätiologie und die Leidenschaft des real)

Die theoretische Arbeit Sigmund Freuds enthält mehrere Verweise auf das Thema der archaischen Erbschaft, welche auch Phylogenie genannt wird. Das Ziel dieser Arbeit ist es, diesen Aspekt seiner Arbeit zu analysieren und die Grenzen der häufigsten Interpretationen aufzuzeigen, welche dazu neigen, den Einfluss des Darwinismus auf Freud zu sehen, oder einfach seine Relevanz zu leugnen. Wir versuchen aufzuzeigen, dass die Einbeziehung der Phylogenie die klinische Frage der Ätiologie der Neurose gänzlich löste.

: Phylogenie, archaisches Erbe, Ätiologie, Geschichte

Citação/Citation: Honorato, S. V., \& Vallejo, M. (2017, setembro). Filogenia y herencia arcaica en la obra de Freud: La búsqueda de la etiologia y la pasion por lo real. Revista Latinoamericana de Psicopatologia Fundamental, 20(3), 544-559. http://dx.doi.org/10.1590/ $1415-4714.2017 \mathrm{v} 20 \mathrm{n} 3 \mathrm{p} 544.9$

Editores do artigo/Editors: Profa. Dra. Ana Maria Rudge e Profa. Dra. Sonia Leite 


\section{ARTIGOS}

Recebido/Received: 8.9.2016 / 9.8.2016 Aceito/Accepted: 10.11.2016 / 11.10.2016

Copyright: (C) 2009 Associação Universitária de Pesquisa em Psicopatologia Fundamental/ University Association for Research in Fundamental Psychopathology. Este é um artigo de livre acesso, que permite uso irrestrito, distribuição e reprodução em qualquer meio, desde que o autor e a fonte sejam citados / This is an open-access article, which permits unrestricted use, distribution, and reproduction in any medium, provided the original authors and sources are credited.

Financiamento/Funding: Os autores declaram não terem sido financiados ou apoiados / The authors have no support or funding to report.

Conflito de interesses/Conflict of interest: Os autores declaram que não há conflito de interesses / The authors have no conflict of interest to declare.

\section{Silvana Vetö Honorato}

Doctora en Historia.

Universidad Andres Bello

Escuela de Psicología, Facultad de Humanidades y Ciencias Sociales

Avda. Fernández Concha 700, Las Condes

Santiago, Chile

silvana.veto@gmail.com

\section{Mauro Vallejo}

Doctor en Psicología.

Universidad de Buenos Aires

Facultad de Psicología

Juan Lavalle 2353

Buenos Aires, Argentina

maurosvallejo@gmail.com

This is an open-access article, which permits unrestricted use, distribution, 